\title{
Gastric Schwannomas Misdiagnosed as GIST: A Comparative Study of Clinic Strategies Based on Membrane Marker Detection
}

\author{
Quanyong Cheng", Kun Zhao\#, Jing Wang\# ${ }^{\#}$ Yu Guo* and Hui Pang*
}

\author{
Departments of Private Medical Center, General Surgery, Obstetrics and Gynecology and Medical Records \\ Management, The First Affiliated Hospital of Sun Yat-sen University, Guangzhou, 510080, China
}

\begin{abstract}
Gastric schwannomas are one of the rarest gastric tumors originating from the nerve plexus of the gut wall. Because most of these tumors don't have any specific symptom and the majority of gastric mesenchymal tumors are gastrointestinal stromal tumors (GISTs), many are therefore misdiagnosed as GISTs. In addition, gastric schwannoma is the benign and slow-growing lesion in the stomach, but GISTs had poor outcomes due to lack of response to nonsurgical interventions. In our study, we analyzed two cases of these tumors. Computer tomography (CT), contrast-enhanced CT, gastroscopy, endoscopic ultrasonography (EUS) were applied to diagnose these two patients. In addition, histological examination and immunohistochemistry (IHC) were used to confirm the final diagnosis. All imageological examination such as CT, contrast-enhanced CT, gastroscopy and EUS, diagnosed these two patients as gastrointestinal stromal tumors. Surprisingly, after the subtotal gastric surgery, histological examination showed that these lesions were composed of spindle cells. Those cells presenting in the bundle or fence-like arrangement were mildly heterologous. The outcomes of immunohistochemistry of the cell membrane markers (CD117 / DOG-1 negative, CD34 mild positive or negative) were the exact opposite of the characteristic presentation of GIST. These pathological findings refused the primary diagnosis, and were in coincidence with the characteristics of gastric schwannomas. To our best knowledge, these tumors are really rare that only two cases could be reported and analyzed clinically. CT and EUS could help diagnose gastric schwannomas before pathological examination results, but in order to define this diagnosis correctly. Pathological examination and IHC staining should be applied after surgery. To avoid the recurrence, it is better to resect the lesion completely, regardless of the malignant or benign disease.
\end{abstract}

Keywords: Gastric Schwannomas, GIST, diagnosis, Cell membrane marker, CD117, CD34, DOG-1, S-100.

\section{INTRODUCTION}

Schwannomas are composed of spindle cells originating from Schwann cells of neural sheath [1]. The stomach is the most common organ for schwannomas in the gastrointestinal tract, and the incidence of gastric schwannomas accounts for $4 \%$ of all benign neoplasms and $0.2 \%$ of all tumors in stomach. The gastric submucosa is the primary site of gastric schwannomas [2]. The neoplasms are often without any symptom, but the most presenting symptom is upper gastrointestinal bleeding and one palpable mass.

Histologically, the typical features of gastric schwannoma include mainly atypical spindle cells arranging in a microtrabecular-microfascicular pattern and a peritumoral lymphoid cuff, with occasional germinal centers [3]. In addition, this disease has the highly unique traits of immunohistochemical positivity for S-100 protein, and negativity for C-kit, CD117, CD34, desmin $[4,5]$.

\footnotetext{
*Address correspondence to this author at the Departments of Private Medical Center and Medical Records Management, The First Affiliated Hospital of Sun Yat-sen University, Guangzhou, 510080, China; Tel: +86-20-87755766; Fax: +86-20-87755766; E-mails: guoyu35@mail.sysu.edu.cn, pangh@mail.sysu.edu.cn

"Equal contributors.
}

However, the morphology and site of gastric schwannomas could often cause difficulties in the differential diagnosis of gastrointestinal stromal tumors (GISTs), which have more malignant potential and need surgical intervention and imatinib-based adjuvant therapy [6]. In general, the detection of specific genetic characteristics, such as a lack of c-Kit and PDGFRa mutations is really helpful to distinguish gastric schwannomas from GISTs [7], and lack of somatic NF2 gene mutations could also be beneficial to differentiate gastric schwannomas from sporadic soft-tissue schwannomas [8].

\section{MATERIALS AND METHODS}

\section{Patient Information}

We analyzed two patients admitted in the Department of Private Medical Center and General Surgery, the First Affiliated Hospital of Sun Yat-sen University. This study was approved by the Clinical Research Ethics Committee of the First Affiliated Hospital of Sun Yat-sen University. Both patients were females, who had signed written informed consents, which enabled them to be eligible for medical ethics [9]. The first patient was admitted with the complaint of upper abdominal pain for ten days. The second patient was admitted because of an abdominal mass revealed by CT scan. Both patients had no history of hypertension, HBV infection or diabetes. 


\section{Laboratory and Imaging Investigations}

After the admission to the hospital, laboratory investigations and imaging procedures including computed tomography (CT) and endoscopic ultrasonography (EUS) were performed for both of the patients. Serum cancer biomarker such as AFP, CEA, CA125, SCC, and CA19-9 were assessed before the surgery [10].

\section{Pathological Examination}

Furthermore, in order to define the final diagnosis, the sections of the lesions obtained through surgical resection were undergone the pathological examination. $\mathrm{HE}$ and $\mathrm{IHC}$ staining were performed to observe the histomorphological features of tumor and to detect the protein expression level of S-100, actin, CD34, CD117, desmin, DOG-1, PDGFRa and Ki67. The procedure adopted for histopathological examination was according to the one described in a study by Guo Y et al. [11].

\section{RESULTS}

\section{Clinical Findings}

The patients underwent the surgical procedures of distant subtotal gastrectomy and Billroth II gastronesteostomy. Laboratory results revealed several serum cancer biomarkers to be within their normal ranges, such as AFP, CEA, CA125, SCC, and CA19-9. Morphologically, in the first patient, a mass measuring $3.5 \mathrm{~cm}$ was found located near antrum of the stomach with intermediate rigidity and clear borders. The gastric serosa was smooth and uninvaded by the lesion. In the second patient the gastric mass was located in the antrum of the stomach with continuous mucosa. No metastatic nodules were found in the surrounding or distant organs; and lymph nodes.

\section{Imageological Findings}

Surgical section from the first patient was evaluated by the non-enhanced computed tomography (CT), which showed a $3.6 \mathrm{~cm} \times 3.0 \mathrm{~cm} \times 3.2 \mathrm{~cm}$ wellcircumscribed mass, with continuous gastric mucosa. On contrast-enhanced CT examination, this tumor displayed homogeneous enhancement, which was strengthened progressively. Besides, near the lesion, there was no swollen lymph node. The diagnosis established by both non-enhanced and contrastenhanced CT was gastrointestinal stromal tumor (Figure 1A, 1B).
The diagnostic gastroscopy revealed one lesion in the submucosa of gastric lesser curvature with smooth surface. In addition, EUS presented a well-defined mass originating from gastric muscularis propria with heterogeneous hypoecho containing sparse blood supply and no swollen lymph node. The diagnosis of gastroscopy and EUS were also gastrointestinal stromal tumor (Figure 1C, 1D).

In the second patient, a $3.2 \mathrm{~cm} \times 3.0 \mathrm{~cm} \times 2.7 \mathrm{~cm}$ mass was identified by the non-enhanced CT showing the image of homogeneous isodense. Contrastenhanced CT was also performed, which showed a mass with unbroken mucosa and swollen lymph nodes near the antrum of stomach. Both of non-enhanced CT and enhanced CT diagnosed this tumor as gastrointestinal stromal tumor (Figure 2A, 2B). Gastroscopy showed a submucosal mass, and EUS also presented a hypoechoic mass with well-defined border adhered to muscularis propria of the stomach. The diagnosis by gastroscopy and EUS were gastrointestinal stromal tumor (Figure 2C, 2D).

\section{Pathologic Findings and Immunohistochemical Findings}

In case 1 , pathologic results showed a round welldefined tough lesion $(3.5 \mathrm{~cm}$ in diameter). After hematoxylin-eosin (HE) staining, microscopic observation displayed that this tumor was located in the gastric muscular layer, composed of spindle cells in the bundle pattern with slight heteromorphism and rare mitosis. The panel of immunohistochemistry (IHC) showed that this tumor was strongly positive for S-100, mild positive for actin, CD34, Ki67\% (5\%), and negative for CD117, desmin, DOG-1, PDGFRa (Figure 3). The pathological diagnosis of this lesion was gastric schwannomas.

In case 2, there shows a solid mass in grey-white and grey-yellow. Microscopic examination showed that this lesion was composed of spindle cells with slight heteromorphism locating in the gastric muscular layer. Large numbers of lymphocytes and plasmocytes were located near the lesion of the stomach. Though the tumor displayed expansive growth, it did not invade the serosa layer. IHC showed that this lesion was positive for S-100, actin, slight positive for Ki67 (2\%), and negative for CD117, CD34, PDGFRa, desmin and Dog1 (Figure 3). Combining the outcomes of microscopic examination and $\mathrm{IHC}$, we got the diagnosis of gastric schwannomas. 
A

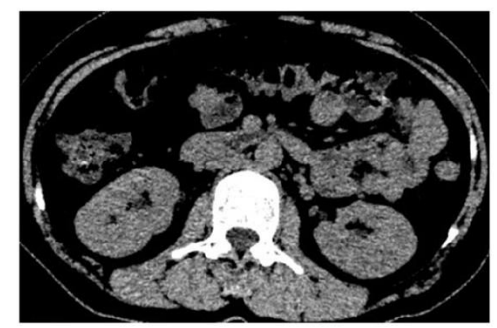

C

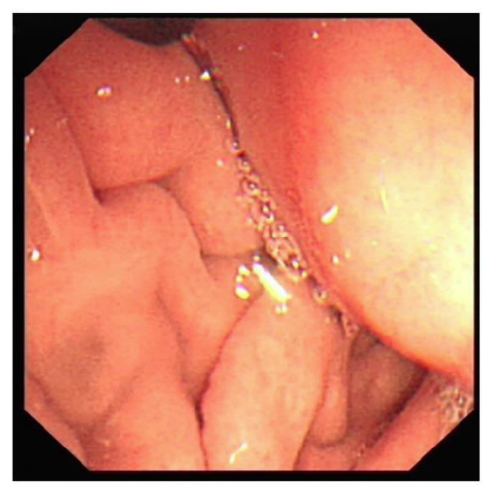

B

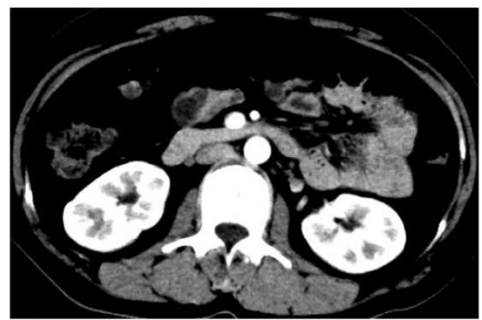

$\mathrm{D}$

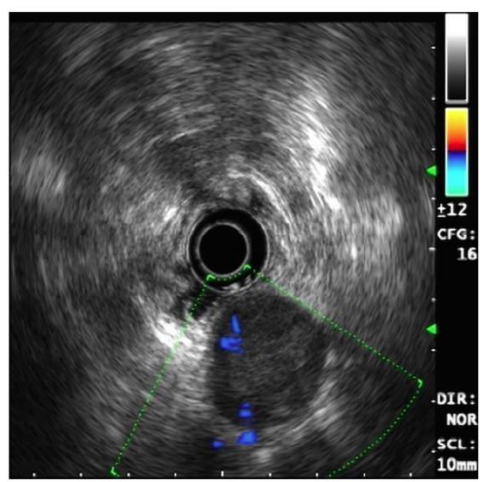

Figure 1: Imageological findings of case 1. A. CT showed a round well circumscribed lesion arising from the lesser curavature of the gastric body. B. Contrast-enhanced CT scan showed moderately enhanced lesion in the arterial phase. C. Gastroscopy presented a round submucosal lesion with smooth surface. D. EUS showed a lesion originating from gastric muscularis propria with heterogeneous hypoechoes.
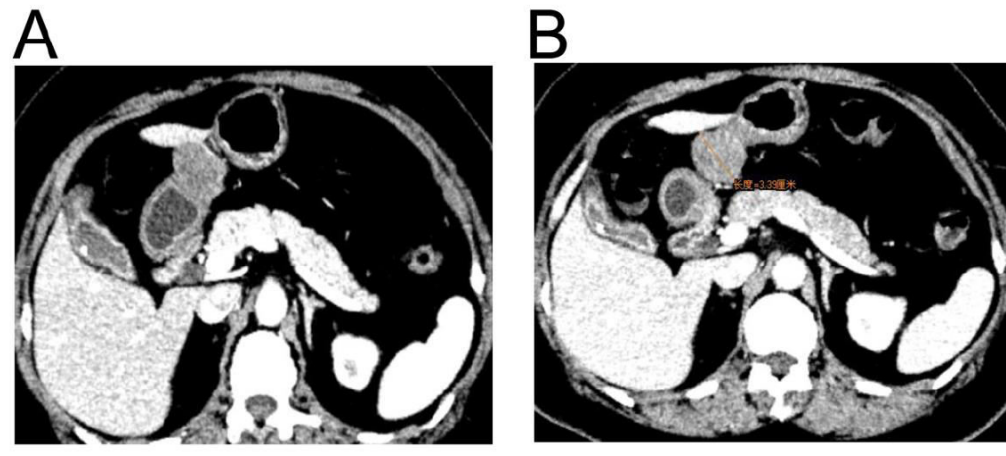

C
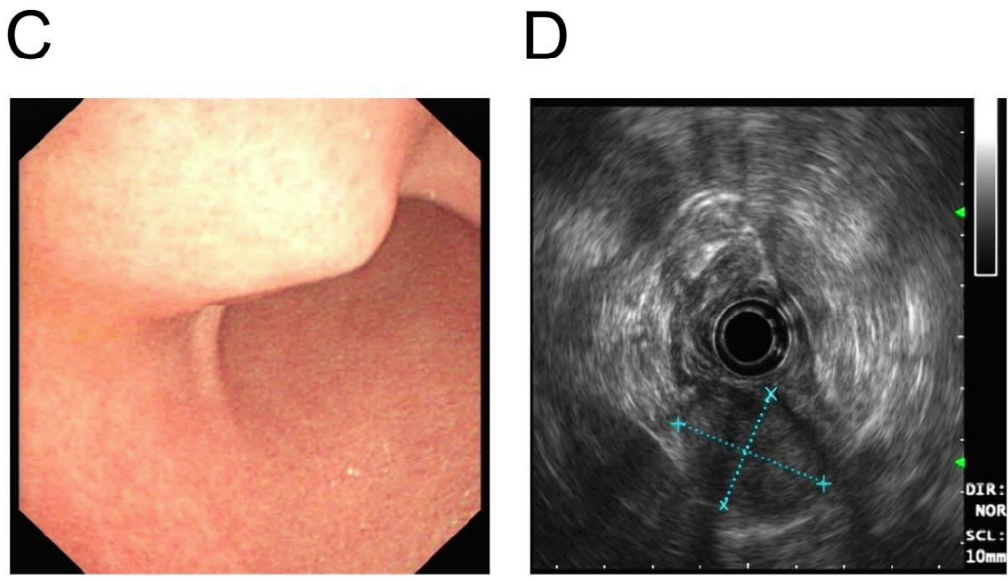

Figure 2: Imageological findings of case 2. A. CT showed the mess of the gastric body. B. Contrast-enhanced CT scan showed enhanced lesion. C. Gastroscopy showed a mass. D. EUS presented a hypoecho mass with well-defined border. 


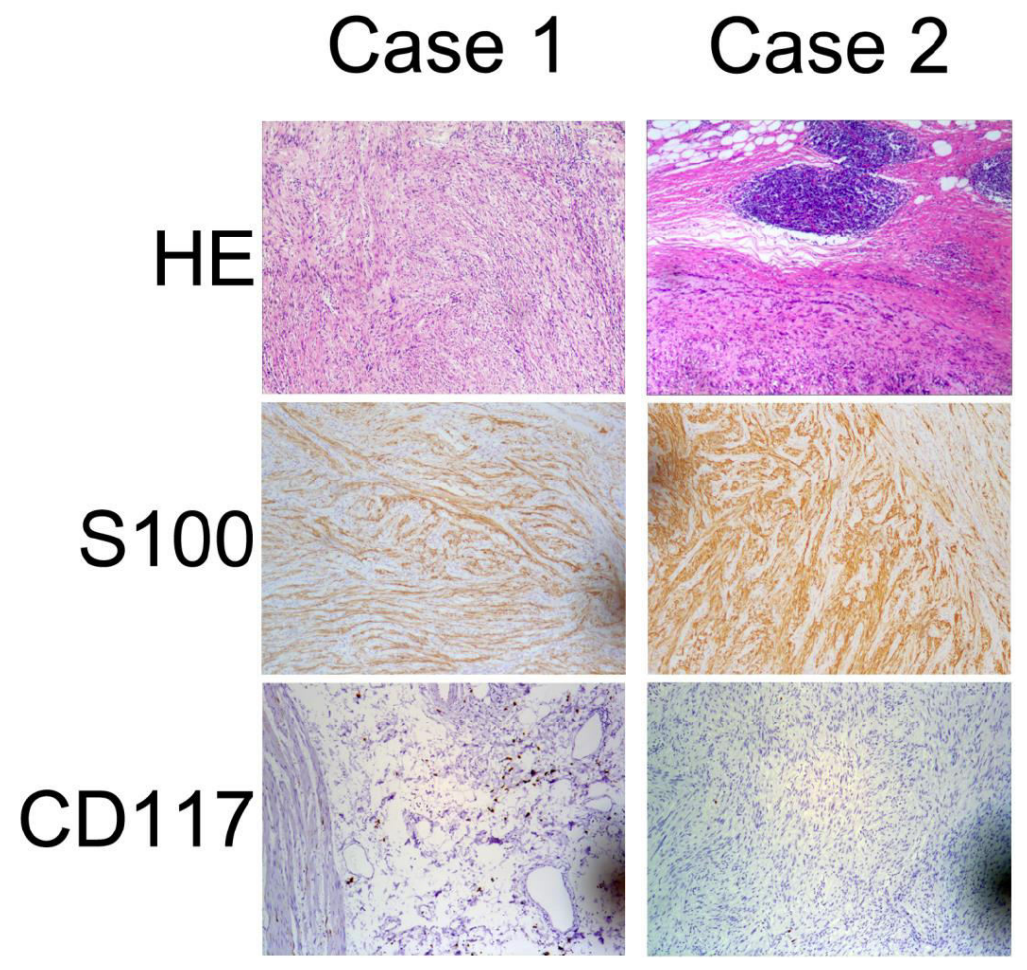

Figure 3: Pathologic findings of patients. Hematoxylin-eosin (HE) staining showed the tissue of the tumor. IHC staining showed the Protein expression level of S100 or CD117.

\section{DISCUSSION}

Gastric schwannomas have rarely been reported due to the low prevalence ranging from $3.3 \%$ to $12.8 \%$ of all mesenchymal tumors [12]. After curative resection, there has been no reported recurrence, metastasis or tumor-related mortality. Several previous studies have reported that gastric schwannomas occur mainly in female patients between the ages of 50 to 60 years old and usually are solitary masses originating from the gastric lesser curvature [1, 13, 14]. Most of them are asymptomatic, or with mild abdominal discomfort. Only in the case of deep ulceration, bleeding might be presented [15]. And when exophytic growth has occurred, a mass may be palpated in the gastric site.

In order to define gastric schwannomas, CT is the most common noninvasive method. On the images of $\mathrm{CT}$, these lesions show the homogeneous appearance and lack degenerative changes, which could enable us to better define leiomyomas from leiomyosarcomas [15]. The previous study showed that most of gastric schwannomas manifested well-defined, ovoid, and homogeneous attenuation without the capsule, hemorrhage, ulceration, or calcification of tumors [16]. Several earlier studies demonstrated that gastric schwannomas have difference from schwannomas of other organs in that they present homogeneous attenuation on CT and that degenerative changes are not common [16, 17]. Gastric schwannomas show a homogenous enhancement pattern on the dynamic CT, and most of these tumors have mild enhancement in the arterial phase and strengthened progressive enhancement in the delayed and venous phases. In addition, the most significant difference between gastric schwannomas and gastrointestinal stromal tumors (GISTs) is that GISTs have more heterogeneous appearance on CT [16]. Furthermore, CT is also very helpful to determine the tumor size and its relation to the surrounding organs.

In addition, MRI is another beneficial technique to identify the location and the tumors' invasion of adjacent vessels and organs [4]. MRI shows the superiority to CT in defining tumor extent, central necrosis and hemorrhage. On MRI examination, these tumors are sharply demarcated and strongly enhanced tumors. Minako Takeda's research showed that gastric schwannomas presented the low signal intensity of T1weighted images and high signal intensity of T2weighted images [18].

Besides, EUS is also a useful tool to make sure the layer of gastric tumor and differentiate the intramural lesions from extrinsic compressions. Previous studies show that the EUS features are round submucosal masses with marginal haloes, and homogeneous 
internal echogenicity with no internal echogenic foci [19]. To differentiate the benign and malignant submucosal tumors, EUS could achieve its value through measuring tumor size and extraluminal margins. But for tumors, which cannot be clearly diagnosed with EUS, EUS-guided fine needle aspiration biopsy (EUS-FNA) and EUS-guided trucut biopsy (EUS-TCB), are better tools for accurate diagnosis [12].

Although it might be useful to get information from CT, MRI, EUS, the definitive diagnosis of gastric schwannomas is from pathological examination. These tumors appear grossly as rubbery, well-circumscribed yellow-white to tan ovoid and range in size from $0.5 \mathrm{~cm}$ to $12 \mathrm{~cm}$ (mean $2.8 \mathrm{~cm}$ ). Histologically, these tumors show the appearance of interlacing woven nests or bundles of spindle cells. Individual cells own eosinophilic cytoplasm without coarse fibrillar material or discernible cell walls, typically with the nuclei being thinner than smooth muscle cell nuclei [20]. The above features can be seen in our cases. From the immunohistochemical aspect, S-100 and actin were rather negative. The two patients in our study were firstly diagnosed of GIST, due to the radiographic and endoscopic investigations. After the surgery, the IHC correctly define the histochemical features. The panel of IHC [21] revealed that S-100 is strongly positive, and CD117, desmin, DOG-1, PDGFRa are negative, proving enough evidence to refuse the diagnosis of GIST and get the finally correct diagnosis.

Gastric schwannomas seem to have good prognosis, with no recurrence, barely metastasizing to lymph nodes. Thus, laparoscopic gastric resection, near-total resection, subtotal resection and wedge resection is the most commonly used treatment, and lymphadenectomy is not routinely performed [22]. In our case, no lymph nodes are found around the lesion. Although the patients with gastric schwannomas could survive well without recurrence and metastasis, most recurrent cases are associated with incomplete surgical margin. Because it's rather difficult to differentiate gastric schwannomas from GIST before pathological examination results, the strategy of treatment for gastric schwannomas is to resect the negative margins and avoid rupture tumors [23].

\section{CONCLUSION}

Our experience confirmed that CT and EUS could help diagnose gastric schwannomas before pathological examination results, but in order to define this diagnosis correctly, we should apply pathological examination and IHC staining after surgery. To avoid the recurrence, it is better to resect the lesion completely, regardless of the malignant or benign disease.

\section{ACKNOWLEDGEMENTS}

We gratefully acknowledge Prof. Yihua Huang and Dr. Jiali Yang for help with the operative procedure and manuscript revision respectively. The datasets supporting the conclusions of this article are available in the First Affiliated Hospital of Sun Yat-sen University. This study was supported by grants from the National Natural Science Foundation of China (81671805, 81302550, 81602723).

We have obtained consent to publish from the participant to report data of every individual patient.

\section{DISCLOSURE OF CONFLICT OF INTEREST}

None.

\section{REFERENCES}

[1] Zheng L, Wu X, Kreis ME, Yu Z, Feng L, Chen C, Xu B, Bu $\mathrm{Z}$, Li Z, Ji J. Clinicopathological and immunohistochemical characterisation of gastric schwannomas in 29 cases. Gastroenterol Res Pract 2014; 2014: 202960. https://doi.org/10.1155/2014/202960

[2] Herzberg M, Beer M, Anupindi S, Vollert K, Kroncke T. Imaging pediatric gastrointestinal stromal tumor (GIST). J Pediatr Surg 2018; 53: 1862-1870. https://doi.org/10.1016/i.jpedsurg.2018.03.022

[3] Tatangelo F, Cantile M, Collina F, Belli A, DE Franciscis S, Bianco F, Botti G. Gastric schwannoma misdiagnosed as GIST: A case report with immunohistochemical and molecular study. Oncol Lett 2016; 11: 2497-2501. https://doi.org/10.3892/ol.2016.4281

[4] Lavy DS, Paulin ET, Parker MI, Zhang B, Parker GS, Schwartz MR. H. Pylori in a gastric schwannoma: a case report. Ann Transl Med 2016; 4: 137. https://doi.org/10.21037/atm.2016.03.55

[5] Bure I, Haller F, Zaletaev DV. Coding and Non-coding: Molecular Portrait of GIST and its Clinical Implication. Curr Mol Med 2018; 18: 252-259.

https://doi.org/10.2174/1566524018666181004113436

[6] Qian XH, Yan YC, Gao BQ, Wang WL. Prevalence, diagnosis, and treatment of primary hepatic gastrointestinal stromal tumors. World J Gastroenterol 2020; 26: 6195-6206. https://doi.org/10.3748/wjg.v26.i40.6195

[7] Lasota J, Miettinen M. Clinical significance of oncogenic KIT and PDGFRA mutations in gastrointestinal stromal tumours. Histopathology 2008; 53: 245-266. https://doi.org/10.1111/j.1365-2559.2008.02977.x

[8] Ogasawara N, Sasaki M, Ishiguro H, Itoh $\mathrm{Y}$, Nojiri S, Kubota E, Wada T, Kataoka H, Kuwabara Y, Joh T. Gastric schwannoma with adjacent external progression harbored aberrant NF2 gene. Dig Endosc 2009; 21: 192-195. https://doi.org/10.1111/j.1443-1661.2009.00885.x

[9] Wang J, Ou J, Guo Y, Dai T, Li X, Liu J, Xia M, Liu L, He M. TBLR1 is a novel prognostic marker and promotes epithelialmesenchymal transition in cervical cancer. $\mathrm{Br} \mathrm{J}$ Cancer 2014; 111: 112-124. https://doi.org/10.1038/bjc.2014.278 
[10] Guo Y, Wang J, Li H, Liu W, Chen D, Zhao K, Liang X, Zhang Q, Yang Y, Chen G. Mediator subunit 23 overexpression as a novel target for suppressing proliferation and tumorigenesis in hepatocellular carcinoma. J Gastroenterol Hepatol 2015; 30: 1094-1103. https://doi.org/10.1111/jgh.12923

[11] Guo Y, Wang J, Zhang L, Shen S, Guo R, Yang Y, Chen W, Wang $Y$, Chen G, Shuai X. Theranostical nanosystemmediated identification of an oncogene and highly effective therapy in hepatocellular carcinoma. Hepatology 2016; 63: 1240-1255. https://doi.org/10.1002/hep.28409

[12] Hong SW, Cho WY, Kim JO, Chun CG, Shim KY, Bok GH, Um WH, Lee JE. Gastric schwannoma diagnosed by endoscopic ultrasonography-guided trucut biopsy. Clin Endosc 2013; 46: 284-287. https://doi.org/10.5946/ce.2013.46.3.284

[13] Yoon W, Paulson K, Mazzara P, Nagori S, Barawi M, Berri R. Gastric schwannoma: a rare but important differential diagnosis of a gastric submucosal mass. Case Rep Surg 2012; 2012: 280982 https://doi.org/10.1155/2012/280982

[14] Shelat VG, Li K, Naik S, Ng CY, Rao N, Rao J, Koura A. Abdominal schwannomas: case report with literature review. Int Surg 2013; 98: 214-218. https://doi.org/10.9738/INTSURG-D-13-00019.1

[15] Williamson JM, Wadley MS, Shepherd NA, Dwerryhouse S. Gastric schwannoma: a benign tumour often mistaken clinically, radiologically and histopathologically for a gastrointestinal stromal tumour--a case series 2012. https://doi.org/10.1308/003588412X13171221590935

[16] Ji JS, Lu CY, Mao WB, Wang ZF, Xu M. Gastric schwannoma: CT findings and clinicopathologic correlation. Abdom Imaging 2015; 40: 1164-1169. https://doi.org/10.1007/s00261-014-0260-4
[17] Rha SE, Byun JY, Jung SE, Chun HJ, Lee HG, Lee JM. Neurogenic tumors in the abdomen: tumor types and imaging characteristics. Radiographics 2003; 23: 29-43. https://doi.org/10.1148/rg.231025050

[18] Takeda M, Amano Y, Machida T, Kato S, Naito Z, Kumita S. $\mathrm{CT}, \mathrm{MRI}$, and PET findings of gastric schwannoma. Jpn J Radiol 2012; 30: 602-605. https://doi.org/10.1007/s11604-012-0093-4

[19] Jung MK, Jeon SW, Cho CM, Tak WY, Kweon YO, Kim SK, Choi YH, Bae HI. Gastric schwannomas: endosonographic characteristics. Abdom Imaging 2008; 33: 388-390. https://doi.org/10.1007/s00261-007-9291-4

[20] Yang LH, Wang JO, Ma S, Zhu Z, Sun JX, Ding SL, Li G, Xu $\mathrm{HT}$, Wang L, Dai SD, et al. Synchronous of gastric adenocarcinoma and schwannoma: report of a case and review of literatures. Int J Clin Exp Pathol 2015; 8: 10411045.

[21] Loewen G, Zhuo Y, Zhuang Y, Jayawickramarajah J, Shan B. lincRNA HOTAIR as a novel promoter of cancer progression. J Can Res Updates 2014; 3: 134-140. https://doi.org/10.6000/1929-2279.2014.03.03.3

[22] Fernandez JA, Frutos MD, Ruiz-Manzanera JJ. Incidenta Gastrointestinal Stromal Tumors (GISTs) and Bariatric Surgery: A Review. Obes Surg 2020; 30: 4529-4541. https://doi.org/10.1007/s11695-020-04853-1

[23] Khobragade KH, Patkar S, Goel M, Sahay A. Primary retroperitoneal GIST: Case report and review of literature. Indian J Cancer 2020; 57: 334-336. https://doi.org/10.4103/ijc.IJC_556_18

\section{DOI: https://doi.org/10.30683/1929-2279.2020.09.09}

(c) 2020 Cheng et al.; Licensee Neoplasia Research.

This is an open access article licensed under the terms of the Creative Commons Attribution Non-Commercial License (http://creativecommons.org/licenses/by-nc/3.0/) which permits unrestricted, non-commercial use, distribution and reproduction in any medium, provided the work is properly cited. 